\title{
Attraction of nonlocal dark optical solitons
}

\author{
Nikola I. Nikolov \\ Informatics and Mathematical Modelling, Technical University of Denmark, 2800 Kongens Lyngby, Denmark, \\ Optics and Fluid Dynamics Department, Risø National Laboratory, \\ OFD-128, P.O. Box 49, 4000 Roskilde, Denmark \\ Dragomir Neshev \\ CUDOS \& Nonlinear Physics Group, Research School of Physical Sciences and Engineering, \\ Australian National University, Canberra, ACT 0200, Australia \\ Wieslaw Królikowski \\ CUDOS \& Laser Physics Center, Research School of Physical Sciences and Engineering, \\ Australian National University, Canberra, ACT 0200, Australia \\ Ole Bang \\ Informatics and Mathematical Modelling and Research Center COM, \\ Technical University of Denmark, 2800 Kongens Lyngby, Denmark \\ Jens Juul Rasmussen \\ Optics and Fluid Dynamics Department, Risø National Laboratory, \\ OFD-128, P.O. Box 49, 4000 Roskilde, Denmark \\ Peter L. Christiansen \\ Informatics and Mathematical Modelling, Technical University of Denmark, 2800 Kongens Lyngby, Denmark
}

We study the formation and interaction of spatial dark optical solitons in materials with a nonlocal nonlinear response. We show that unlike in local materials, where dark solitons typically repel, the nonlocal nonlinearity leads to a long-range attraction that enables the formation of stable bound states of dark solitons.

(C) 2003 Optical Society of America

OCIS codes: $190.5530,190.4420$

Dark solitons, which have an intensity profile in the form of a dip in an otherwise uniform background, are topological objects because of their nontrivial phase structure. It appears that dark solitons always repel ${ }^{1}$, unless special external perturbations are imposed. Already early theoretical studies of the self-defocusing nonlinear Schrödinger (NLS) equation indicated the repulsive nature of dark soliton interaction ${ }^{2}$. The first systematic investigations were conducted by Zhao and Bourkoff ${ }^{3}$, who numerically studied the propagation of closely placed dark temporal solitons in optical fibers and found that their interaction was repulsive and weak compared to bright solitons. Subsequent experimental studies of temporal ${ }^{4}$ and spatial dark solitons ${ }^{5-7}$ confirmed that their repulsive interaction is generic.

To suppress the repulsion of dark solitons, Afanasjev et al. perturbed the Kerr response of a nonlinear medium by incorporating higher order gain terms ${ }^{8}$. Ostrovskaya et al. proposed the use of "solitonic gluons" 9 , i.e., two out-of-phase weak bright beams guided by closely spaced dark solitons.

Here we discuss propagation of dark solitons in a nondissipative self-defocusing nonlocal Kerr-like medium.
We show that the nonlocality drastically modifies the interaction of dark solitons by inducing a long-range attraction between them, thereby enabling the formation of stable dark soliton bound states for the first time in a homogeneous medium without external perturbations.

We consider the evolution of a one-dimensional optical beam $u=u(x, z)$ described by the nonlocal NLS equation

$$
\begin{aligned}
& i \partial_{z} u+\partial_{x}^{2} u+\Delta n(I) u=0, \\
& \Delta n(I)=-\int_{-\infty}^{\infty} R(x-\tau) I(\tau) d \tau,
\end{aligned}
$$

where $I=I(x, z)=|u|^{2}$ is the intensity, $z$ is the evolution coordinate, $x$ represents a transverse coordinate, and $\Delta n$ is the nonlinear refractive index change of the medium. The function $R(x)$ characterizes the nonlocal response of the medium and is assumed to be real and symmetric.

The nonlocal term $\Delta n(I)$ in Eq. (1) represents a general phenomenological model for self-defocusing Kerrlike media, in which the change in the refractive index induced by an optical beam involves a transport process. This may include diffusion of molecules or atoms in atomic vapours ${ }^{10}$ or thermal effects in plasma ${ }^{11}$. A 


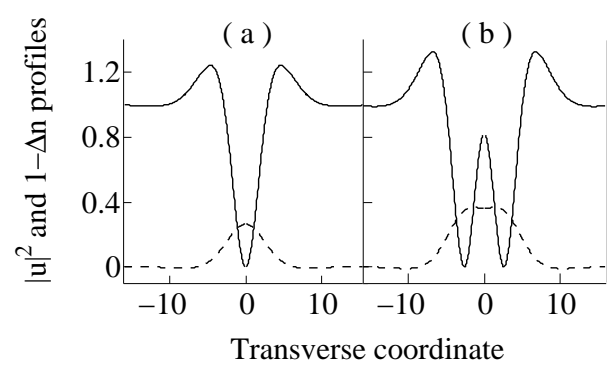

Fig. 1. Intensity profile $|u(x)|^{2}$ (solid line) and induced waveguide structure: $1+\Delta n(x)$ (dashed line) of a single nonlocal dark soliton (a) and a bound state (b) for $\lambda=1$. The degree of nonlocality is $\sigma=4$.

nonlocal response in the form (1) appears naturally due to many body interaction processes in the description of Bose-Einstein condensates ${ }^{12}$. It was shown recently that such a nonlocal nonlinearity prevents collapse of multidimensional beams and supports formation of bright solitons ${ }^{13-15}$.

Without loss of generality ${ }^{16,17}$ we consider the normalized response function $R(x)=(2 \sigma)^{-1} \exp (-|x| / \sigma)$. This form allows us to write Eq. (1) as two coupled equations

$$
\begin{aligned}
& i \partial_{z} u+\partial_{x}^{2} u+\Delta n u=0, \\
& \Delta n-\sigma^{2} \partial_{x}^{2} \Delta n=-|u|^{2},
\end{aligned}
$$

where Eq. (3) is the diffusion equation for a defocusing material. In this context the degree of nonlocality $\sigma$ is then the diffusion parameter. In particular, for $\sigma \rightarrow 0$ the model (1) approaches the local Kerr nonlinearity.

Let us focus on fundamental dark solitons $u(x, z)=$ $u(x) \exp (i \lambda z)$, where the real profile $u(x)$ has zero center amplitude, $u(0)=0$, and a $\pi$ phase jump at the center, and where $\lambda$ is a propagation constant. It was shown recently ${ }^{18-20}$ that Eqs. (2-3) written for these stationary dark solitons are identical to the equations describing spatial solitons in quadratic nonlinear (or $\chi^{(2)}$ ) materials. The $\chi^{(2)}$ system predicts ${ }^{21}$ the existence of stationary dark solitons with monotonic tails for $\sigma<1 / \sqrt{8}$ and nonmonotonic tails for $\sigma>1 / \sqrt{8}$. This implies that bound states of two or more dark solitons can be formed in sufficiently nonlocal materials with $\sigma>1 / \sqrt{8}$.

In Fig. 1 we show a numerically found dark soliton solution and the corresponding two-soliton bound state for $\sigma=4$. The dashed line shows the soliton-induced waveguide $(1+\Delta n)$, in which the soliton is guided.

It is important to note that although the profiles of stationary nonlocal spatial dark solitons and their bound states are equivalent to those of the $\chi^{(2)}$ system, the $\chi^{(2)}$ dark soliton bound states are unstable and breakup when propagating, while the corresponding nonlocal bound states are stable, robust entities. We demonstrate the stability by numerical integration of Eq. (1) with the exact numerically found solution as initial condition. Our simulations confirm stable propagation of nonlocal dark
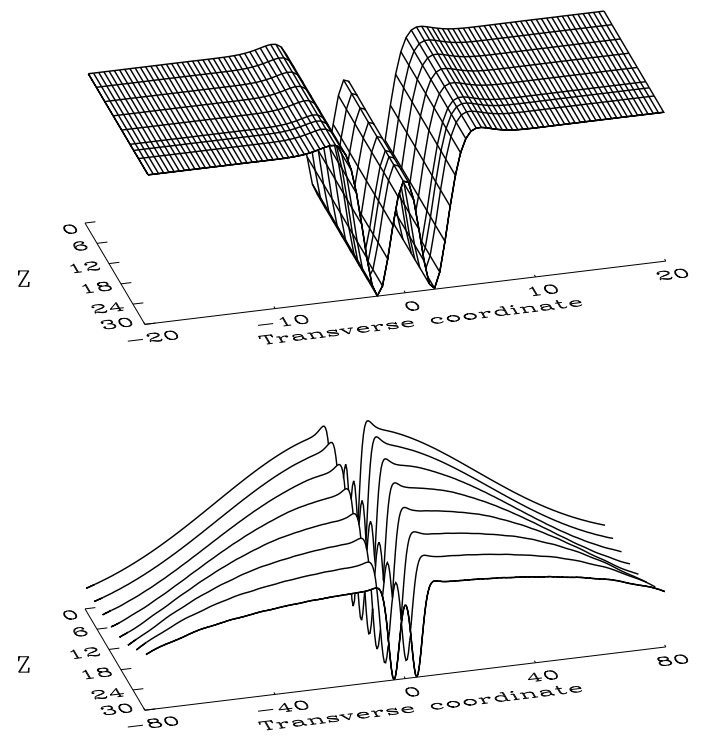

Fig. 2. Evolution of the intensity profile of a bound state of two nonlocal dark solitons for $\sigma=1$ and $\lambda=1$ on a cw background (top) and a Gaussian background (bottom).

solitons and their bound states over tens of diffraction lengths, as the example shown in Fig. 2.

For a better verification of stability we used the same initial condition, but now embedded in a broad Gaussian beam, as in typical experiments. The propagation of this structure is shown in Fig. 2(b). The solitons remain bounded even though the background beam experiences strong deformation due to the self-defocusing. As the intensity of the background beam decreases the solitons adiabatically follow, adjusting their widths and mutual separation.

The ability of nonlocal dark solitons to form stable bound states is a direct consequence of the long-range attraction induced by the nonlocality. This effect may be explained using the self-guiding concept. In a local defocusing Kerr medium the refractive index distribution corresponding to two dark solitons always has the form of two waveguides separated by a region of lower refractive index which acts as a potential barrier. This potential barrier leads to their repulsion. In the presence of a sufficiently strong nonlocality, for which the width of the response function $(\sigma)$ is comparable to or larger than the separation between the solitons, the convolution integral in Eq. (1) is able to even out this central dip completely and create a single broad waveguide (see Fig. 1), which traps the solitons and enable them to form a bound state.

Dark solitons are generated experimentally using odd or even boundary condition ${ }^{5,6}$. In the first case a broad laser beam passes through a phase-mask with a single phase jump, leading to formation of an odd number of dark solitons. In the second case the beam passes through an amplitude mask (a thin wire), which imposes a dark 
22. V.E. Zakharov and A.B. Shabat, Sov. Phys. JETP 37, 823 (1972). 